\title{
VULNERABILIDAD HOSPITALARIA E IMPACTO EN LA SALUD DE LOS TRABAJADORES POST TRASLADO AL HOSPITAL DE CAMPAÑA DEL HOSPITAL REGIONAL DE ICA 2008-2011
}

"Hospital vulnerability and I impact in the health of the workers post transfer to field Hospital Ica Regional Hospital 2008-2011

Dora Isabel Pino Arana ${ }^{1,2, a, b, c, d}$

\footnotetext{
${ }^{1}$ Facultad de Enfermería "Universidad Nacional San Luis Gonzaga" de Ica. Ica, Perú

${ }^{2}$ Hospital Regional de Ica, Ica, Perú

${ }^{a}$ Licenciada en Enfermería, ${ }^{b}$ Especialista en cuidados Intensivos y Centro Quirúrgico ${ }^{c} \mathrm{Mg}$. En salud Publica, ${ }^{d}$ Doctor en medio ambiente y desarrollo sostenible,
}

\section{RESUMEN}

Objetivo: Determinar la vulnerabilidad hospitalaria e impacto en la salud de los trabajadores post traslado al Hospital de Campaña del Hospital Regional de Ica 2008-2011. Material y métodos.- El nivel descriptivo aplicativo retrospectivo. Se aplicó un modelo matemático que determinó la vulnerabilidad hospitalaria, instrumento validado por la OPS estos tomaron en cuenta los componentes; estructurales, no estructurales y funcionales, para lo cual fue necesario evaluar 145 aspectos o elementos del hospital. Para evaluar el impacto en la salud del personal del hospital se realizaron preguntas estructuradas, cerradas y fáciles de responder, las cuales fueron dirigidas a indagar las afecciones sobre salud respiratorias, de piel, digestivas, renales, psicosociales .RESULTADOS Se tomó una muestra de 130 trabajadores y el impacto sobre la salud hallándose moderada $66.1 \%$, la seguridad estructural fue de alta en un $74 \%$, en el componente no estructural fue de bajo grado de seguridad en un $73 \%$, y en el componente funcional fue de un $71 \%$. Los componentes de la seguridad hospitalaria se correlacionaron indirecta y significativamente con el impacto en la salud de los trabajadores. Conclusiones: Existió vulnerabilidad hospitalaria post traslado al Hospital de Campaña del Hospital Regional de Ica 2008-2011, siendo el componente no estructural y funcional las más vulnerables frente a una emergencia o desastre. El impacto en la salud de los trabajadores está presente.

Palabras Claves: Vulnerabilidad hospitalaria, Impacto, salud, de los trabajadores.

\section{SUMMARY}

Objectives: To determine the hospital Vulnerability and I impact in the health of the workers post transfer to the campaign Hospital Ica 2008-2011. Material and methods.- The level used is the descriptive retrospective application, wich was used in this investigation ,the predominant type of analysis is quantitative, but qualitative scoring and interpretations on the mutual correlation between the study variables. we applied a mathematical model that determines the vulnerability hospital by PAHO validated instrument for the rapid diagnosis of low-cost and easy to apply for health personnel, scores were admitted to an Excel spreadsheet (mathematical model) and automatically tabuled the results, they took into account the structural, nonstructural and functional for which it was necessary to evaluate 145 areas of the hospital which hurtled like in one of the three categories of high hospital security, she mediates, CONCLUSIONS: The present work reaches the conclusion that the hospital vulnerability is of stocking to high post transfer to the campaign hospital 2008-2011. Being not the component structural and functional the most vulnerable of responding appropriately in front of an emergency or disaster. Of equal it forms the impact in the health of the workers.

Keywords: Vulnerability hospital, Impact , health of workers. 


\section{INTRODUCCIÓN.}

Un hospital seguro es un establecimiento de salud cuyos servicios permanecen accesibles y funcionando a su máxima capacidad y en su misma infraestructura inmediatamente después de que ocurre un desastre natural que amerite la emergencia y el afrontamiento al desastre como evento adverso. El término abarca a todos los establecimientos de salud, cualquiera sea su nivel de complejidad. Es seguro, porque cuenta con la máxima protección posible, asimismo las vías de acceso al establecimiento de salud y los servicios de suministro de agua potable, energía eléctrica y telecomunicaciones continúan operando, lo que permite garantizar su funcionamiento continuo y absorber la demanda adicional de atención médica (1).

El presente trabajo de investigación tiene por finalidad determinar la vulnerabilidad hospitalaria e impacto en la salud de los trabajadores post sismo en el Hospital Regional de Ica 2008-2011. Asimismo, se plantea como objetivos específicos determinar la vulnerabilidad hospitalaria post traslado al Hospital de Campaña del Hospital Regional de Ica 2008-2011, en todos sus componentes y su correlación con el impacto en la salud de los trabajadores.

Se planteó un trabajo de investigación aplicada; de nivel descriptivo utilizando el método de investigación científica MIC y métodos secundarios como el análisis, síntesis, inductivo, deductivo, observacional y estadístico con diseño correlacional.

El muestreo propuesto es de tipo probabilístico al 95\%, utilizándose un modelo matemático y la aplicación de un cuestionario como instrumento.

Consta de tres capítulos, habiéndose estructurado el trabajo de acuerdo al método de la investigación científica actual. En la primera se detalla el planteamiento metodológico, en el segundo el marco teórico y en el último los resultados encontrados.

Considerando el rigor metodológico detallado en el presente trabajo se puede concluir que constituye un importante documento de consulta para toda persona interesada en la investigación.

\section{MATERIAL Y METODOS.}

Tipo de Investigación aplicada, Nivel de Investigación descriptiva, método de Investigación Científica (MIC), proceso de investigación cuyo resultado es la ciencia; así como la estrategia de la misma.

Los métodos secundarios son: Análisis, síntesis, inductivo, deductivo, observacional y estadístico. Diseño Correlacional. La población estuvo compuesta por todos los trabajadores del hospital regional que fueron 412 trabajadores. Muestra constituida por 130 trabajadores asistenciales los cuales están conformadas por: médicos, enfermeras, obstétricas, odontólogos, técnicos de enfermería y laboratorio, entre otros. En la recolección de datos se utilizó como técnica la encuesta dirigida averiguar los estados de salud de los trabajadores del hospital Regional de Ica en el 2008 y 2011., y como instrumento para determinar la vulnerabilidad hospitalaria se utilizó el índice de seguridad hospitalaria este es un instrumento validado por la Organización Panamericana de La Salud (OPS) para el diagnóstico rápido de bajo costo y fácil de aplicar por el personal de salud, los puntajes fueron ingresados en una hoja electrónica de Excel (modelo matemático) y automáticamente se tabularon los resultados, estos tomaron en cuenta los componentes estructurales, no estructurales y funcionales para lo cual fue necesario evaluar 145 aspectos o elementos del hospital el cual arrojó como en una de las tres categorías de seguridad hospitalaria alta, media, o baja (2).

Para evaluar el impacto en la salud del personal de salud del hospital se realizaron preguntas estructuradas cerradas fáciles de responder las cuales fueron dirigidas a indagar las afecciones de salud respiratorias, de piel, digestivas, renales, psicosociales y otros. 


\section{RESULTADOS.}

Se tomó una muestra de 130 trabajadores de los cuales el $60,8 \%$ de sexo femenino y $39,2 \%$ de sexo masculino, de los cuales el $29,2 \%$ fueron médicos, el $24,6 \%$ técnicos de enfermería, 23,9\% enfermeras, técnicos de nutrición y de limpieza el $4,1 \%$ y con respecto a promedio de edades el $31,6 \%$ están comprendidas entre 31 y 40 años. De los cuales el $72,3 \%$ manifestaron aumento de estrés, depresión en el $60 \%$ y afección económica en el 51,6\%. En cuanto a los trastornos lumbares se encontró un 36,1\%, siendo la enfermedad renal la menos frecuente $11,5 \%$ y las de la piel $23,8 \%$. Luego del traslado post terremoto al Hospital de Campaña, el impacto sobre la salud ha sido moderada en un $66,1 \%$, el $20,8 \%$ de menor impacto y en un $13,1 \%$ de los casos un impacto mayor. (Tabla 1) Al determinar el índice de seguridad del hospital, se halló una vulnerabilidad de 0,54 y un índice de seguridad de 0,46 , la determinación evaluativa parcial del componente de seguridad estructural fue de alta en un $75 \%$, mientras que un $25 \%$ se categoriza como de seguridad media (tabla 2).

En el componente no estructural fue de bajo grado de seguridad en un $73 \%$, en un $16 \%$ como alto $11 \%$ como seguridad media (tabla 3) y en el componente funcional fue de un $71 \%$ bajo, $20 \%$ categorizado como grado medio y $9 \%$ seguridad alta (tabla 4) Los componentes de la seguridad hospitalaria se correlacionaron indirecta y significativamente con el impacto en la salud de los trabajadores.

\section{TABLA 1. IMPACTO EN LA SALUD DE LOS TRABAJADORES DEL HOSPITAL REGIONAL DE ICA}

\begin{tabular}{lccc}
\multicolumn{2}{c}{ IMPACTO EN LA SALUD DE LOS } & \multicolumn{2}{c}{ FRECUENCIAS } \\
\cline { 2 - 4 } & TRABAJADORES & $\mathbf{N}^{\circ}$ & $\%$ \\
\hline MAYOR & 17 & 13,1 \\
MODERADO & 86 & 66,1 \\
MENOR & 27 & 20,8 \\
\hline TOTAL & $\mathbf{1 3 0}$ & $\mathbf{1 0 0 . 0}$ \\
\hline
\end{tabular}

TABLA 2. VULNERABILIDAD HOSPITALARIA SEGÚN EL GRADO DE SEGURIDAD ESTRUCTURAL

\begin{tabular}{lccc} 
& \multirow{2}{*}{ SEGURIDAD ESTRUCTURAL } & \multicolumn{2}{c}{ FRECUENCIAS } \\
\cline { 2 - 4 } & $\mathbf{N}^{\circ}$ & $\%$ \\
\hline ALTO & 109 & 75 \\
MEDIO & 36 & 25 \\
BAJO & 0 & 0 \\
\hline TOTAL & $\mathbf{1 4 5}$ & $\mathbf{1 0 0 . 0}$ \\
\hline
\end{tabular}

TABLA 3. VULNERABILIDAD HOSPITALARIA SEGÚN EL GRADO DE SEGURIDAD NO ESTRUCTURAL

\begin{tabular}{lccc} 
& \multirow{2}{*}{ SEGURIDAD NO ESTRUCTURAL } & \multicolumn{2}{c}{ Frecuencias } \\
\cline { 2 - 3 } & $\mathbf{N}^{\circ}$ & $\%$ \\
\hline ALTO & 23 & 16 \\
MEDIO & 16 & 11 \\
BAJO & 106 & 73 \\
\hline TOTAL & $\mathbf{1 4 5}$ & $\mathbf{1 0 0 , 0}$ \\
\hline
\end{tabular}

TABLA 4. VULNERABILIDAD HOSPITALARIA SEGÚN EL GRADO DE SEGURIDAD FUNCIONAL

\begin{tabular}{lccc} 
& \multirow{2}{*}{ SEGURIDAD FUNCIONAL } & \multicolumn{2}{c}{ Frecuencias } \\
\cline { 2 - 3 } & $\mathbf{N}$ o & $\%$ \\
\hline ALTO & 13 & 9 \\
MEDIO & 29 & 20 \\
BAJO & 103 & 71 \\
\hline TOTAL & $\mathbf{1 4 5}$ & 100,0 \\
\hline
\end{tabular}




\section{DISCUSIÓN.}

El presente estudio se realizó considerando la vulnerabilidad hospitalaria y el impacto en la salud de los trabajadores del Hospital de campaña. Para lo cual se tomó una muestra de 130 pacientes encontrándose el $60,8 \%$ de sexo femenino, el $29,2 \%$ son médicos y el $31,6 \%$ tienen sus edades comprendidos entre 31 y 40 años, el $72,3 \%$ de los trabajadores encuestados manifestaron aumento de estrés, seguido de depresión con el $60 \%$ y la afección económica con el 51,6\%. La enfermedad que resalta en frecuencia fueron las enfermedades lumbares representado por el $36,1 \%$ siendo la enfermedad renal la menos frecuente $(88,5 \%)$ y las de la piel $(75,4 \%)$. Luego del traslado post terremoto al Hospital de campaña, El impacto en la salud del $66,1 \%$ de los encuestados ha sido moderada, en el $20,8 \%$ de los casos ha sido menor y en un $13,1 \%$ de los casos han tenido un impacto mayor en su salud.

Al determinar el índice de seguridad del hospital, que toma en cuenta el medio ambiente y la red de servicios de salud a los que pertenece, se tiene un índice de vulnerabilidad de 0,54 y un índice de seguridad de 0,46, ésta última clasifica al establecimiento según las recomendaciones base en la categoría " $\mathrm{B}$ " en donde se requiere medidas necesarias en el corto plazo, ya que los niveles actuales de seguridad existentes pueden potencialmente poner en riesgo a los pacientes, el personal y su funcionamiento durante y después de un desastre. Mientras que en la investigación realizada por Rodrigo Restrepo Gonzales (5), se encontró que el índice de vulnerabilidad fue de 0,46 y un índice de seguridad de 0,54 medida en el Hospital San Francisco de Asís de Quibdó en el país vecino de Colombia. Su determinación evaluativa parcial del componente de seguridad estructural fue de alta en un $74 \%$, similar al determinado en el Hospital Regional de campaña (Ica, Perú) que resultó en un Alto grado de seguridad de $75 \%$ apropiado para la capacidad de respuesta en salud. Su determinación evaluativa parcial del componente de seguridad no estructural del Hospital San Francisco de Asís (6) dio una calificación de medio en un $51 \%$ y bajo en un $28 \%$, mientras que el determinado en el Hospital Regional de campaña fue de Bajo grado de seguridad en un $73 \%$, mientras que en un $16 \%$ fue alto y en un $11 \%$ fue medio, lo cual nos permite definir que ante situaciones de emergencia y desastres, la respuesta del hospital Regional podría verse afectado debido a las fallas que pudieran presentarse en este aspecto o componente. Por último de acuerdo al aspecto de seguridad funcional evaluada por el autor fue de baja en un $75 \%$ y medio en un $21 \%$, y la encontrada en el Hospital de campaña es de Bajo grado de seguridad en un $71 \%$ y medio en un $20 \%$, lo cual permite definir que este componente al igual que la no estructural son la mayor debilidad del Hospital de campaña, ya que es altamente probable que en casos de emergencia y desastre la respuesta a víctimas y afectados no sea lo más apropiado debido al evento adverso (7). Los componentes de la seguridad hospitalaria se correlacionan indirecta y significativamente con el impacto en la salud de los trabajadores.

\section{CONCLUSIONES.}

1. Existe Vulnerabilidad hospitalaria post traslado al hospital de campaña del hospital regional de Ica 2008-2011. Siendo los componentes no estructural y funcional los más vulnerables.

2. El impacto en la salud de los trabajadores post traslado al hospital de campaña es de moderada a mayor 2008-2011.

3. El valor de $r=-0,5480$ determina que la vulnerabilidad hospitalaria del tipo estructural se correlaciona moderadamente con el impacto en la salud de los trabajadores post traslado al Hospital de campaña de Ica 2008-2011.

4. El valor de $r=-0,5720$ determina que la vulnerabilidad hospitalaria del tipo no estructural se correlaciona modera- 
damente con el impacto en la salud de los trabajadores post traslado al Hospital de campaña de Ica 2008-2011.

5. El valor de $r=-0,7620$ determina que la vulnerabilidad hospitalaria del tipo funcional se correlaciona altamente con el impacto en la salud de los trabajadores post traslado al Hospital de campaña de Ica 2008-2011.

\section{RECOMENDACIONES.}

Se recomienda a las autoridades del Hospital regional que se socialice el plan de respuesta hospitalario para emergencias y desastres con la finalidad de estar preparados ante la ocurrencia de eventos adversos naturales y producidos por el hombre para ejecutar acciones inmediatas con oportunidad, eficiencia y eficacia y de esta manera reducir las vulnerabilidades no estructurales $y$ funcionales. Es fundamental que se mejore el componente funcional de la seguridad hospitalaria, así como garantizar que funcione el Comité Hospitalario de Emergencia, su normalización mediante acto administrativo, mediante el cual se conforma y elaborar un plan que deberá establecer todos los procesos, procedimientos y accionar organizativo para la respuesta de los trabajadores ante eventos adversos sin que tenga un mayor impacto en su salud.

\section{Correspondencia:}

\section{Isabel Pino Arana}

Correo electrónico: jjjjisa@hotmail.com

\section{REFERENCIAS BIBLIOGRAFICAS}

1. OPS/OMS. Hospitales seguros; 2008 [fecha de acceso 12 de junio de 2011]. URL Disponible en: http://helid.digicoll ection.org/pdf/s8284s/s8284s.pdf

2. OPS/OMS. Guía de la OMS y la OPS para el uso de hospitales de campaña extranjeros en caso de desastres. [fecha de acceso 17 de junio de 2011]. URL disponible en: http://www.disasterinfo.net/hospital disaste r/assets/HospitalesDeCampana.pdf
3. OPS. Hospitales de Campaña; 2003. [fecha de acceso 2 de julio de 2011]. URL disponible en: http://helid.digicollection. org/en/d/Js8254s/4.html

4. Respetro R. Medición del índice de seguridad hospitalaria en el Hospital San Francisco de Asís de Quibdó; Colombia, 2009.

5. Pereyra E., Elías C., Morales N. Vulnerabilidad sísmica Hospitalaria: Experiencias del Hospital Regional de Ica a cuatro años del desastre. Ica; 2011.

6. OPS/OMS. Fundamentos para la mitigación de desastres en establecimientos de salud; 2004.

7. OPS/OMS. Índice hospitalario. [Fecha de acceso 22 de julio de 2011]. URL disponible en: http://safehospitals.info/ index.php?option=com content\&task=view \&id=30\&ltemid $=1$ 